\title{
DEPSIM: NUMERICAL 3D-SIMULATION OF THE WATER, GAS AND SOLID PHASE IN A LANDFILL
}

\author{
S. SCHMUCK ${ }^{1}$, D. WERNER ${ }^{2}$, R. WIDMANN ${ }^{1} \&$ T. RICKEN ${ }^{2}$ \\ ${ }^{1}$ University of Duisburg-Essen, Germany. \\ ${ }^{2}$ Technical University of Dortmund, Germany.
}

\begin{abstract}
The model depSIM is a dump simulation model, which allows a detailed and time-scaled focus into the complex processes of a landfill. Description of the mechanical model: The biological, chemical and physical processes in the waste body are closely connected with each other and can be described mechanically. Therefore, a number of differential equations are needed and implemented in the model. The porous media body is examined under the acceptance of a compressible gas phase, a materially incompressible solid state, an organic phase and a liquid phase. For the verification of the numerical model the long-time behaviour (100 years) was simulated. Further details about the model and the mechanical background are summarized in Robeck, Ricken et Widmann: A finite element simulation model of biological conversion processes in landfills [1]. Use potentials: The developed model allows a differentiated, time wise and locally calculation and representation of the temperature, the organic conversion rate, the local pressure ratios and the gas current speeds. There were several case studies with the depSIM model in Germany which show the correlation between the temperature, gas production and gas potential. Therefore three different landfills were evaluated. Here, in the correlation between measured temperature in the landfill body and the temperature in the model was shown. The average divergence between both was less than 2 degree. By the detailed calculation of the gas speeds in every point of the dump an essential improvement arises compared with conventional arithmetic models for gas forecast and gas capture. These forecast models are based on estimated initial parameters. This allows only forecasts for a complete dump or a dump segment, but allows no coupled calculation of the relevant parameters. The model depSIM offers a spatially differentiated consideration of the gas production. However, just a spatially exact, quantitative forecast of the gas production is necessary for dump operator and authorities. The right forecast is elementary for the right dimensioning of the gas collection system and gas treatment and the possible use in combined heat and power units. All gas streams can be shown with the simulation model along the dump surface spatially and time wise differentiated. This allows a locally differentiated dump gas management with a division in areas with active or passive gas collection or to estimate the feasibility of a methane oxidation layer.

Keywords: biological degradation, gas collection, gas production, landfill, methane oxidation, methane production, simulation, waste disposals.
\end{abstract}

\section{INTRODUCTION}

Worldwide, the most common way of waste disposal are landfills. After solid waste is deposited in a landfill, different physical, chemical, and biological processes take place which modify the waste. The most important is the biological degradation of the biological fixed carbon. Due to these reactions, landfill gas is produced inside the landfill body and effuses into the atmosphere. These processes create environmentally harmful landfill gases with concentrations of about $45 \%-65 \%$ methane $(\mathrm{CH}-4)$ and $35 \%-55 \%$ carbon dioxide $\left(\mathrm{CO}_{2}\right)$ and further tracer gases. The main substances cause the greenhouse effect. 
Unfortunately, the impact of methane is 21-25 times higher than that of carbon dioxide. Therefore, it is essential to estimate the long-term behaviour of a landfill.

During the operation phase of a landfill, the accounting for gas production, settlement, and waste water are important.

If the production of methane is high, the active gas suction and the use in combined heat and power units is state-of-the-art in developed countries. Over time, methane production decreases to the point that active care of the landfill becomes economically, as well as technically, inefficient. Thus, a passive methane oxidation layer could help decrease harmful emissions. These layers contain methane reducing bacteria that convert methane into carbon dioxide.

\section{BASIC MODEL: THEORY OF POROUS MEDIA AND MIXTURE THEORY}

The description of complex and complicated processes or material behaviour is done by computers. These calculations are based on models, which are able to describe the considered processes.

For the calculation of these different processes of municipal solid waste landfills a suitable theoretical model has to be developed which is able to describe the complex transport and conversion processes within a landfill.

The Theory of Porous Media (TPM) has been developed for the description of multi phase systems. First implementations were done in soil mechanics to describe the behaviour of the considered three phase system composed of a solid, fluid and a gas phase. Further applications can be found in biomechanics for the description of growth processes or to describe the effects of freezing thawing cycles on materials like asphalt or concrete. The TPM allows a detailed description of the mentioned processes and is performed successfully on these areas. The difference between each application area is the constitutive modelling which describes the specific material behaviour. The idea was to use the concept of the TPM for the description of the behaviour of landfill bodies because landfills are also multiphase systems composed of several phases. For a realistic description of the transport and conversion processes within a landfill the constitutive modelling had to be adapted. Herein, the landfill body is considered as a four phase system composed of two solid phases (inorganic and organic phase) and two fluid phases (liquid and gas phase).

\subsection{Theory of porous media}

The TPM is the mixture theory classified by the concept of volume fractions. Hereby, we consider a continuum which consists of several constituents. The investigated porous body consists of S (solid), O (organic), L (liquid) and G (gas), see Fig. 1.
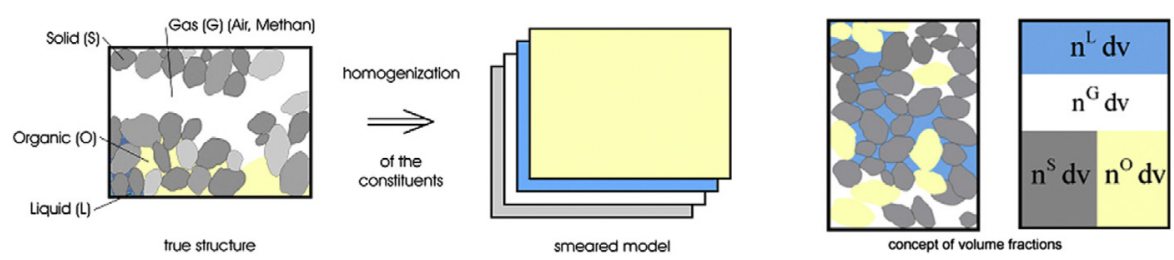

Figure 1: Homogenization of the true structure and concept of volume fractions [1]. 
In the volume fraction concept, it is assumed that the porous solid always models a control space and that the pores are statistically and homogeneously distributed [2].

A porous medium occupying the control space of the porous solid, consists of constituents ( $\mathrm{a}=\mathrm{S}, \mathrm{O}, \mathrm{L}, \mathrm{G}$ ) with real volumes. The boundary is a material surface for the solid phase and a non-material surface for the liquid and gas phases.

The volume fraction concept contains the volume fractions, which refer the volume elements of the individual constituents to the bulk volume element [3].

Due to the volume fraction concept, all geometric and physical quantities, such as motion, deformation and stress, are defined in the total control space.

Thus, they can be interpreted as the statistical average values of the real quantities.

\subsection{Assumptions}

For a manageable scope, the following assumptions were made. The liquid phase is incompressible. The two solid phases are incompressible as well, and they have the same motion. The assumption of the incompressible matter is based on the compressibility (of the mixture body) due to the poro-elasticity is much higher than the compressibility due to the true phase compression. The settlements (displacements) in a landfill are caused by the changing porosity due to load and to the biological conversion process.

Even the solid and the liquid phase are not involved in the mass transition.

The last assumption is based on a negligible temperature exchange between all the phases in each time step. This assumption does not work under a high temperature difference between the phases. In this application case for different German landfills such high temperature gradients are not existent.

\subsection{Constitutive modelling}

Municipal solid waste and other disposed wastes consist of a complex mixture of inorganic and organic matters. The biological degradation by microorganism is limited on the organic content of the waste and on the influence of inhibitory substances. The mineral part of the waste is not involved in biological conversion processes. The assumption of no mass transition for the inorganic solid phase is consecutive. The organic content is divided into a biological degradable part and a part that is nearly non-degradable. The non-degradable parts are plastics. The biologic degradable organic materials are divided into three fractions (rapid, moderate and slow).

The biological degradation is done by several groups of microorganisms, which are specialized on specific substrates and are dependent on specific milieu conditions.

A general classification can be made into aerobic and anaerobic conditions. After a short aerobic period where the available oxygen in the landfill body the acidification and instable methane phase follows. These periods usually take between $1 / 2$ and 2 years.

In the following so-called stabile methane phase with a gas composition of about $55 \%-65 \%$ of methane and $35 \%-45 \%$ of carbon dioxide and a constant neutral $\mathrm{pH}$ value between 7 and 8 .

This stable methane phase is the dominant phase during the life span of a landfill and it is calculated by the depSIM model. Herein, the organic mass supply expresses the changes of the organic phase due to bacterial activity into the gas phase. This conversion process with its degradation kinetics is variable in place and time and depends on several influencing factors. The main influence factor for the biological degradation is the temperature. Therefore, Robeck [4] implemented the following three different temperature stages in the depSIM Model (Fig. 2). 

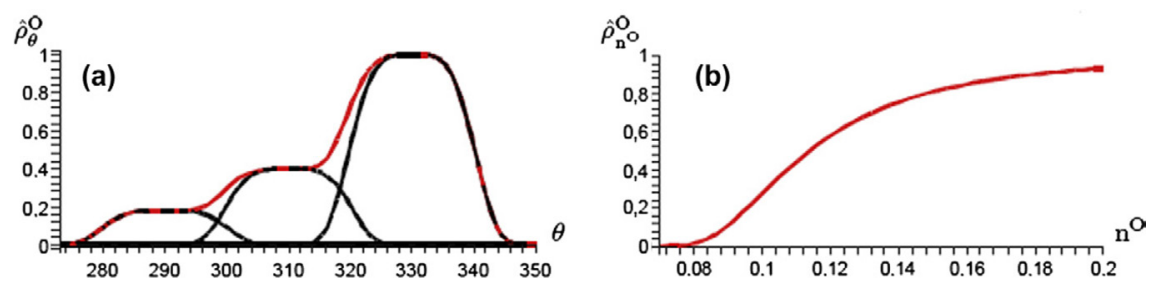

Figure 2: Main influencing factors on the maximum degradation rate max: (a) temperature (b) organic content [4].

\section{SIMULATION PROCESS}

Before starting the simulation, annual data need to be collected for the amount and composition of the deposited waste and the location of the waste deposition over time. Annual surveys with topographic information about the landfill construction from the beginning of deposition in combination with lists about the deposited waste amount for each year are necessary to reconstruct the genesis and geometry of the landfill precisely. If waste density is unknown, then it can be calculated by the combination of annual surveys (volume) and deposited waste amount (mass) combined with data from additional literature research. The knowledge about the annual deposited waste types (domestic waste, biowaste, market waste, demolition waste, sludges, inert waste, etc.) is important to estimate the organic carbon content. Local variations of the deposited waste composition over time have to be considered. With these information, the landfill can be described with its individual genesis (geometry). This is essential for a detailed description of the conversion processes.

After the data processing, the landfill geometry has to be discredited by a finite element mesh and boundaries for the process parameters have to be set. In the depSIM model the landfill structure is considered annually while the time step for the simulated processes is 1 month. The calculation has been made with four- node tetrahedral elements. The elements and nodes have to be made individually for each landfill. Based on the boundary conditions, the gas phase leaves the control space at the upper boundary. Free heat exchange and air temp of $290 \mathrm{k}$ is assumed as boundary conditions for the boundary landfill/air. The initial volume fraction of the organic phase is annually specified between 0.16 and 0.18 with an individual waste composition. The real density is assumed to be $1,000 \mathrm{~kg} / \mathrm{m}^{3}$.

\section{APPLICATION}

The first step of simulation of a landfill is discredited by a finite element mesh. In Fig. 3, the finite element mesh of a 25 ha landfill is shown. It consists of 14,551 nodal points with 76,592 elements. The required time with an eight-core computer for the calculation is 1.5 weeks.

Figure 4 shows a 3D-Model of the landfill, which allows a detailed view of the temperature distribution. DepSim points out high temperatures (e.g. $>400$ Kelvin) in the core on the system, where a biological degradation is not effective or not possible. By the detailed calculation of the gas speeds at every point of the dump a summing up is possible. An essential improvement compared with conventional arithmetic models for gas forecast and gas capture is implemented. These forecast models are based on estimated initial parameters. This allows only forecasts for a complete dump or a dump segment, but not for specific elements. 


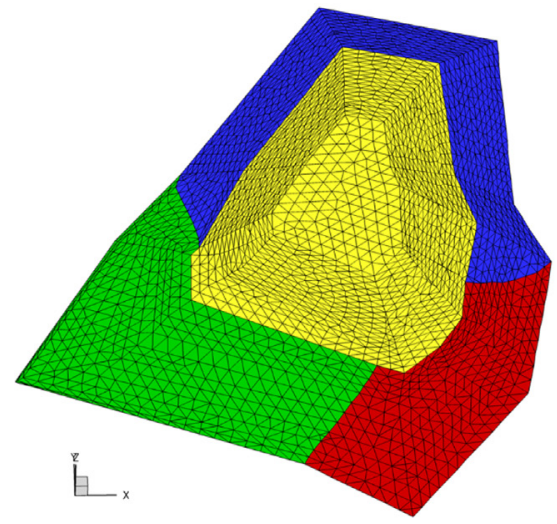

- disposal area I (1978 - 1983)

- disposal area II (1983 - 1987)

- disposal area III (1987 - 1993)

- disposal area IV (1994 - 2003)

Figure 3: Finite Element Mesh of a landfill.

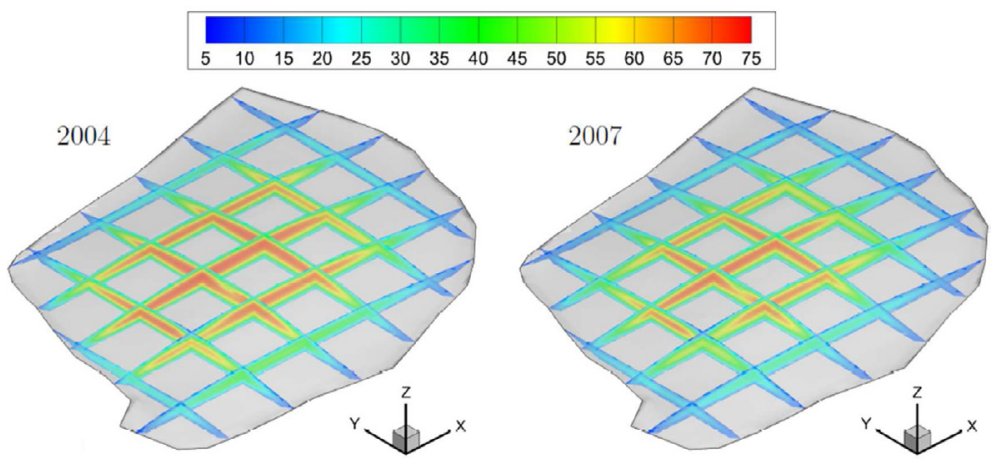

Figure 4: Simulation of a landfill body [1].

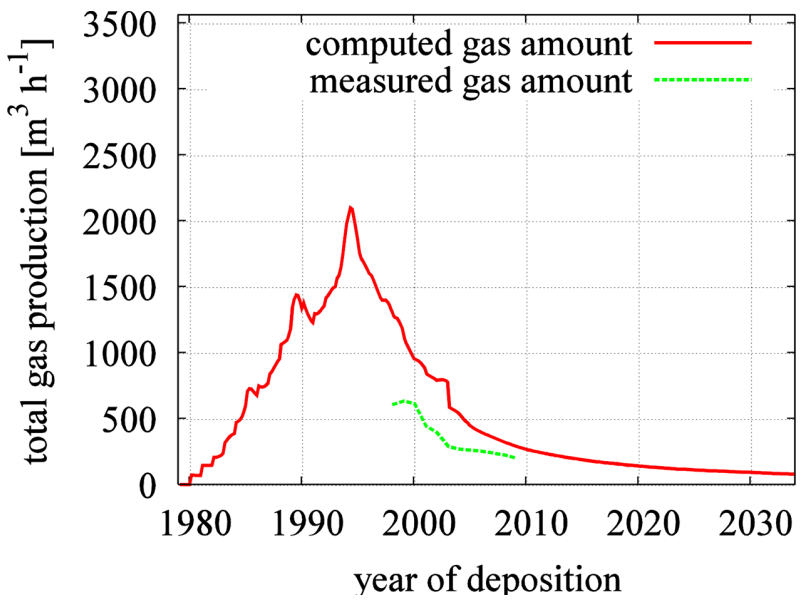

Figure 5: Comparison of measured and computed gas production. 


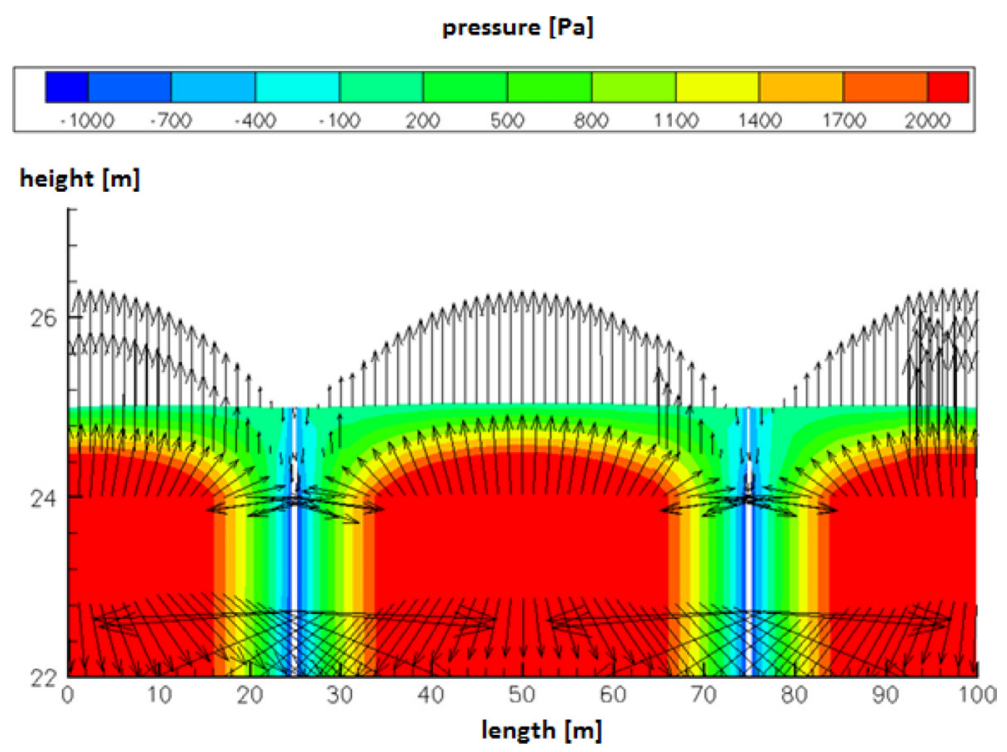

Figure 6: Simulation of a two gas well system.

The depSIM model was used to simulate the gas production for the landfill. In Fig. 4, a comparison between the measured gas and the computed gas amount is shown. A similar progress of both is clearly recognizable.

The model depSIM offers a spatially differentiated consideration of the gas production. Figure 6 shows the pressure distribution in a landfill with two gas wells. DepSim computes the amount of collected gas and the surface current.

However, just a spatially exact, quantitative forecast of the gas production is necessary for dump operators and authorities. The right forecast is elementary for the right dimensioning of the gas collection system and gas treatment and the possible use in combined heat and power units. All gas streams can be shown with the simulation model along the dump surface spatially and time-wise differentiated. This allows a locally differentiated dump gas management with separated areas with active and passive gas collection or to estimate the feasibility of a methane oxidation layer.

\section{REFERENCES}

[1] Robeck, M., Ricken, T. \& Widmann, R., A multiphase finite element simulation of biological conversion processes in landfills. Waste Management, 31, pp. 663-669, 2011. http://dx.doi.org/10.1016/j.wasman.2010.08.007

[2] Ricken, T. \& Ustohalova, V., Modeling of thermal mass transfer in porous media with applications to the organic phase transition in landfills. Computational Material Science, 32, pp. 498-508, 2005. http://dx.doi.org/10.1016/j.commatsci.2004.09.015

[3] Ricken, T. \& de Boer, R., Multiphase flow in a capillary porous medium. Computational Material Science, 28, pp. 704-713, 2003.

http://dx.doi.org/10.1016/j.commatsci.2003.08.032

[4] Robeck, M., A FEM Simulation of Transport and Conversion Processes in Landfills using a Multiphase Model Based on the Theory of Porous Media, Shaker Verlag: Aachen, 2012. 